\title{
Visiting a Physiotherapist is Useful in the Early Phase of Low Back Pain
}

\section{Karvonen $\mathrm{E}^{*}$}

Department of sport and health sciences, University of Jyvaskyla, Finland

*Corresponding author: Eira Karvonen, University of Jyvaskyla, Uimakallionkatu 4, Fin15170 Lahti, Finland, Tel: +358 40 5076405; Email: eira.karvonen@kolumbus.fi

\section{Research Article}

Volume 3 Issue 2

Received Date: July 27, 2020

Published Date: August 13, 2020

DOI: $10.23880 /$ aphot-16000157

\section{Abstract}

Background: The purpose was to examine physiotherapists' (PTs) core competences in direct access practice for low back pain (LBP) in the early phase of pain using tissue-structural classification, and the implementation of this practice after a continuing education with clients' and PTs' experiences.

Methods: Although previous research has shown direct access in physiotherapy to be an important method in preventing the recurrence of LBP, data are still lacking on the level of required competences of PTs, classifications of LBP and implementation of direct access practice after a continuing education program.

Results: PTs' core competences of clinical reasoning and critical reflection were on a good level when used tissue-structural classification and hypothetico-deductive method. PTs made accurate diagnostic subgroups for their LBP clients. The agreement between PTs and reviewers was 74\% (kappa 0.63; 95\% CI, 0.47 to 0.77). PTs also analyzed their clinical reasoning systematically from clients' history to choices of clinical tests and conclusions. They justified their manual skills and the adequacy of the LBP classification in use and suggested other alternatives for their performance. $80 \%$ of the clients were satisfied with the implementation of direct access in the early visit to PTs and with the information and advice they received. Three months after the physiotherapy, repeat visits were on a low level and only one of the employed was on sick leave. PTs reported their work meaningful which, according to a qualitative analysis, was connected with clients` satisfaction, reasonable division of labor, cooperation with their co-workers and an increase in professional appreciation.

Conclusions: This research demonstrates the benefits, as outlined by clients and PTs, of direct access to physiotherapy in the early phase as well as the use of tissue-structural classification (nociceptive pain mechanism) as one of the classifications in this stage. Clinical reasoning and critical reflection competences in the assessment of clients were important during the sub classification and during the evaluation of PTs' own performance. Continuing learning of these competences should also be included in education programs. These results can be utilized in PTs' direct access practice as well as when planning the content of the education program.

Keywords: LBP-Classification; Clinical Reasoning; Critical Reflection; Core Competences; Direct Access 


\section{Introduction}

Low back pain (LBP) and related dysfunctions are the second leading cause of early disability and high health care costs in Finland. The recommendations of clinical guidelines for early clinical examination of LBP clients, avoiding unnecessary imaging and surgery, rapid restoration of work capacity and support for returning to work, have contributed to the possibility of people with LBP and other musculoskeletal disorders to seek direct physiotherapists instead of visiting a doctor. On the basis of the agreed screening questionnaire, clients directly applying to the physiotherapist (PT) and those in need of medical attention will be booked by persons trained for it. The possibility for doctor's rapid consultation allows time assignment directly to a PT already, for example online [1-3].

\section{Aims}

The purpose of this study was to examine PTs' competences in the assessment of LBP clients and the implementation of direct access practice after a continuing education program. More specifically the aims were to investigate the following:

- How accurately do PTs apply their clinical reasoning in sub classification of LBP clients when using tissuestructural classification of LBP?

- How do PTs justify their clinical reasoning and how critically do PTs reflect on their clinical reasoning in their assessment of LBP clients?
- What are clients' experiences of the implementation of PTs' direct access practice?

- What are PTs' experiences of the implementation of their direct access practice?

\section{Methods}

This thesis is based on four studies with different designs, where both quantitative and qualitative approaches were used as well as on the analysis of clinical reports written by PTs, questionnaires and interviews.

Prior to the start of direct access practice, additional training was carried out for physiotherapists in the health centers in South-Finland, where the content of the training was based on knowledge of the different pain mechanisms and the practical selection and implementation of suitable or appropriate pain classifications. The training also included the identification of signs of serious diseases, assessment of the need for consultation with a doctor, on the basis of symptomatic and findings of examination, and assessment of psychosocial stress factors, e.g. through a written questionnaire [4,5].

Figure 1 shows the classifications of LBP at different stages of the duration of pain. For early LBP (= less than 3 months) it was directed to use the classifications in the tissue and structure level of pain and movement control with treatment instructions.

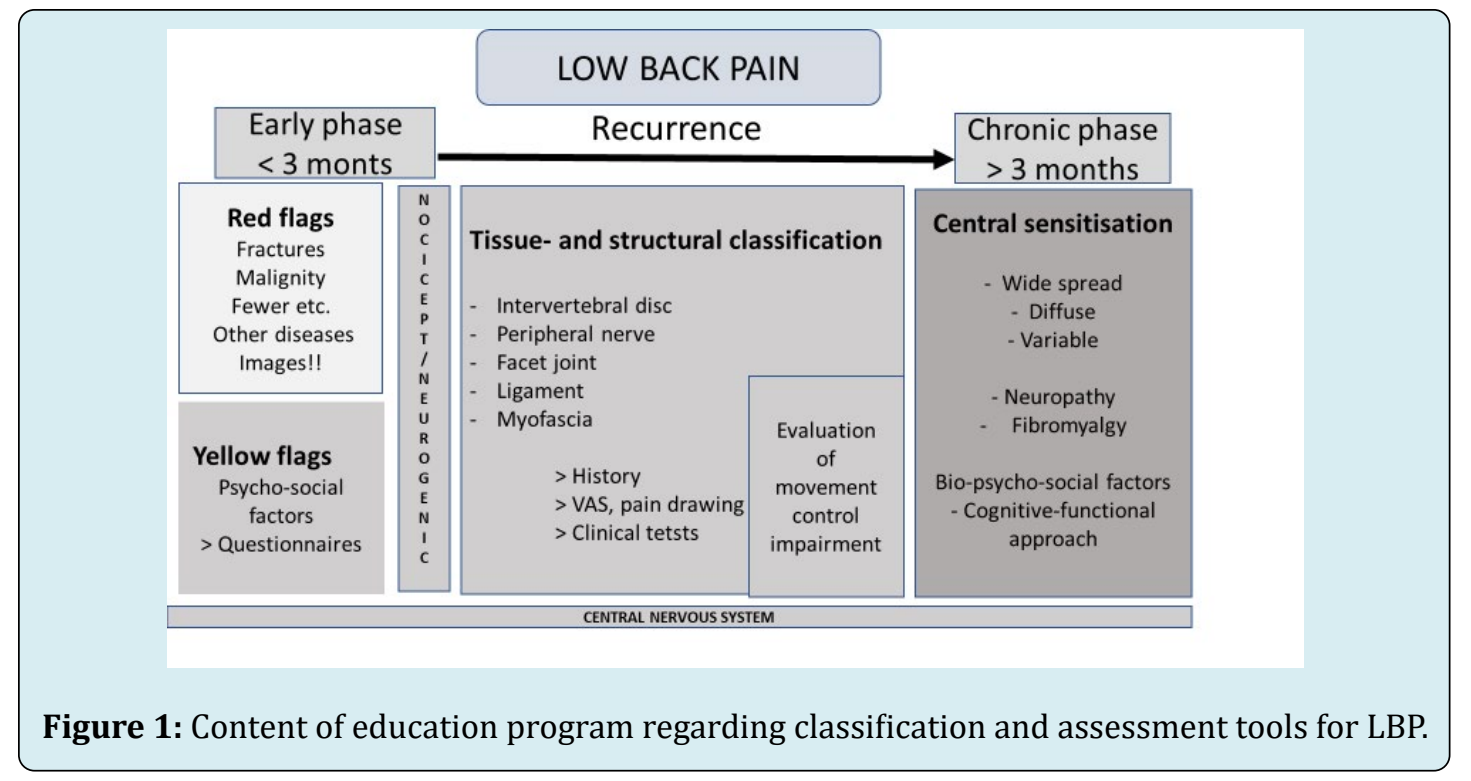

When interviewing the clients, it was also instructed to recognize the clients' life situation and its effects on experiencing pain and the suitability of the instructions, utilizing the various elements of the international functional classification (ICF) from general health to environmental and personality factors (Figure 2). 


\section{Annals of Physiotherapy \& Occupational Therapy}

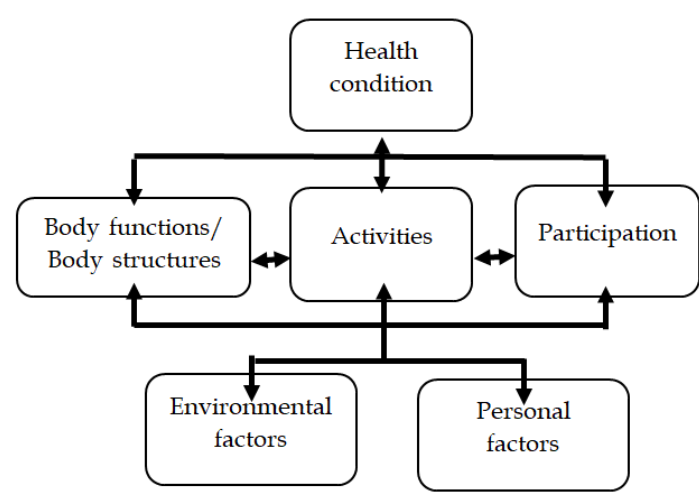

Figure 2: Bio-psycho-social model of the International Classification of Functioning, Disability and Health (ICF).

\section{Results}

The results can be summarized and observed from two perspectives. The first is from that of PTs' competences (clinical reasoning competence in the assessment of LBP client with tissue-structural classification of LBP and critical reflection competence in clinical reasoning). The second is from the perspective of the implementation of direct access practice (clients' and PTs' experiences).

\section{Clinical Reasoning and Critical Reflection Competence}

PTs were able to apply the clinical reasoning competence with tissue-structural classification and to make consistently accurate diagnostic decisions about subgroups for clients with LBP in its early phase. The level of agreement between the decisions of the two reviewers and PTs showed $74 \%$ overall agreement (kappa $=0.63,95 \% \mathrm{CI}: 0.47-0.77$ ) of all client subgroups; (Table 1).

\begin{tabular}{|c|c|c|c|c|}
\hline LBP classification & 6 PTs & 2 Reviewers & Agreement\% & Kappa (95 \% CI) \\
\hline Discogenic pain & 3 & 5 & 60 & \\
\hline $\begin{array}{c}\text { Clinical instability / segmental movement control } \\
\text { impairment }\end{array}$ & 13 & 10 & 77 & \\
\hline $\begin{array}{c}\text { Clinical / functional lumbar spinal stenosis } \\
\text { Segmental dysfunction / facet pain }\end{array}$ & 0 & 1 & 0 & \\
\hline Sacroiliac joint pain/dysfunction & 10 & 11 & 91 & \\
\hline Other classification & 6 & 3 & 33 & \\
\hline Not classified & 2 & 4 & 25 & \\
\hline Overall agreement & & & 74 & \\
\hline Kappa & & & & $0.63(0.47$ to 0.77$)$ \\
\hline
\end{tabular}

Table 1: Inter examiner agreement of the classification of 57 clients with low back pain between PTs and reviewers.

PTs critically analyzed and reflected on their choices and decisions on clinical reasoning regarding client's symptoms, onset of pain, clinical test choices and test results using the hypothetic-deductive reasoning model in their conclusions. Special attention was given to clients with recurrence of pain or with psychosocial stress factors.

\section{Clients' and PTs' Experiences}

More than three quarters (80\%) of clients in all three subgroups (retired, unemployed and employed, and student group) were told to get help for their back pain a lot or moderately (Figure 3). Clients viewed the early visit to a physiotherapist as useful in the treatment of back pain and in the prevention of new pain episodes. A notable observation was that only one person was on sick leave during the previous three months. The results also showed some differences between different client groups even if benefits from direct access visits seemed to occur in all groups.

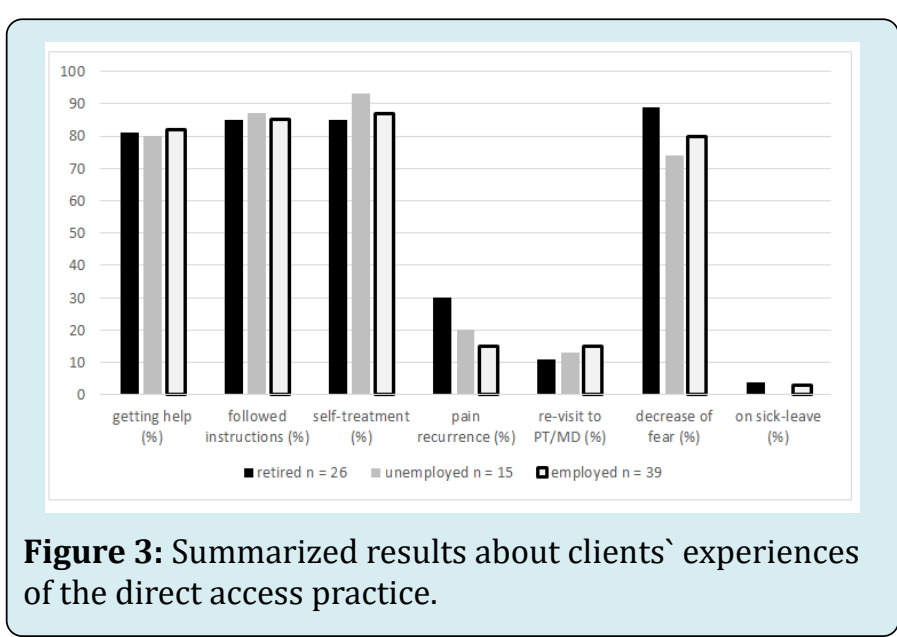




\section{Annals of Physiotherapy \& Occupational Therapy}

PTs described their experiences of direct access practice from three perspectives: clients perspective, the work perspective and working communities perspective (Figure 4).

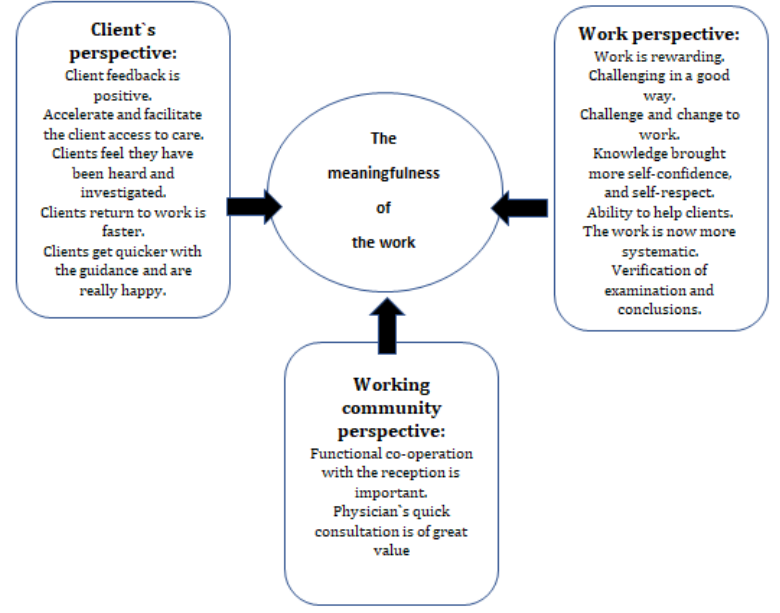

Figure 4: Summarized results about physiotherapists experiences of their direct access practice.

In clients' perspective PTs highlighted clients' satisfaction with direct access by fast access to care, with guidance of their back pain, and quick return back to work. In work perspective, PTs expressed the growth of professional pride, self-confidence and appreciation. They experienced their work now more systematic. In work communities` perspective, PTs commented the importance of functional co-operation with reception, with physicians and other health care professionals.

In connection with these three perspectives, PTs felt the increase of the meaningfulness of their work. PTs indicated numerous aspects also to be essential: the possibility to regularly update their knowledge and information, training new staff members as well as having a sufficient amount of PTs to implement the new practice.

The summarized results regarding PTs experiences of their direct access practice has been described in Figure 4.

\section{Discussion}

When observing PT"s clinical reasoning competence, the results showed that PTs were able to use tissue-structural classification of LBP and hypothetico-deductive reasoning in the examination of their clients when observing PTs subgroup conclusions with this classification. Hypotheticodeductive reasoning has been used when a nociceptive pain mechanism is related to the symptoms. PTs also critically reflected on their choices and decisions. For example, their discussed the inadequacy of the classification for clients with psychosocial stress factors. It has been noted the need for LBP classification according to nociceptive pain mechanism and movement dysfunction, but it has also pointed out that all pain implies activation of the brain, including activity in regions responsible for cognitive-emotional and affective progressing input. So even if results of these dissertations showed that PTs could use tissue-structural classification in examination of their clients in good level it has also shown the need to evaluate clients' psycho-social stress factors to understand clients pain experience and influences for their movement dysfunctions. Both clients and PTs satisfaction with direct access practice give positive input to continue and develop this practice.

The results of this dissertation are similar to those carried out elsewhere in PTs' direct access. This activity benefits not only clients, but also society as the cost of health care decreases. So far, no situation is known where patients safety has been compromised in this practice. Apparently, PTs have been able to assess when the expertise of a doctor or other health care person is needed to determine the client’s condition.

As a further study of physiotherapists' direct access practice, there will be a longer follow up for the preservations of the results and possible causes of pain in larger group of clients in several medical care districts in Finland.

\section{Conclusions}

This research demonstrates the benefits, as outlined by clients and physiotherapists, of direct access to physiotherapy in the early phase of LBP as well as the use of tissuestructural classification (nociceptive pain mechanism) as one of the classifications in this stage. Clinical reasoning and critical reflection competences in the assessment of clients were shown to be important during the sub classification of LBP clients and during the evaluation of physiotherapists' own performance. Continuing learning of these competences should also be included in education programs. The results of this research can be utilized in physiotherapists' direct access practice as well as when planning the content of the education program for the practice.

\section{References}

1. Karvonen E, Paatelma M, Kesonen JP, Heinonen A (2015) Knowledge translation from continuing education to physiotherapy practice in classifying patients with low back pain. Journal of Manual and Manipulative Therapy 23(2): 68-74. 


\section{Annals of Physiotherapy \& Occupational Therapy}

2. Karvonen E, Paatelma M, Laitinen Väänänen S, Piirainen A (2017) Clinical reasoning and critical reflection in physiotherapists' examinations of patients with low back pain in its early phase: A qualitative study from physiotherapists' point of view. European Journal of Physiotherapy 19(4): 185-193.

3. Karvonen E, Paatelma M, Heinonen A (2017) Patients' experiences of their visit to direct access physiotherapists in the early phase of low back pain: A retrospective descriptive survey. Kuntoutus 40(3-4): 3-11.

4. Karvonen E, Laitinen Vaananen S, Paatelma M, Roine M, Heinonen A (2019) Physiotherapists' experiences of direct access for clients with musculoskeletal pain and dysfunction: a qualitative study. European Journal of Physiotherapy.

5. Karvonen E (2020) Physiotherapy for Low Back Pain Clients in Direct Access Practice: Competences and Implementations. pp: 189. 